\title{
Long-term effects of an exercise and Mediterranean diet intervention in the vascular function of
}

\section{an older, healthy population.}

Markos Klonizakis ${ }^{\mathrm{a}}$, Ahmad Alkhatib ${ }^{\mathrm{b}}$, Geoff Middleton ${ }^{\mathrm{c}}$

${ }^{a}$ Centre for Sports and Exercise Science,Sheffield Hallam University, UK,

m.klonizakis@shu.ac.uk

${ }^{\mathrm{b}}$ Academy of Sport and Physical Activity, Sheffield Hallam University, UK, a.alkhatib@shu.ac.uk

c School of Sport and Exercise Science, University of Lincoln, UK, gmiddleton@lincoln.ac.uk

Keywords: microcirculation, fitness assessment, nutrition, endothelium, ageing, Mediterranean diet

Correspondence: Dr. Markos Klonizakis

Centre for Sports and Exercise Science

Sheffield Hallam University

Collegiate Crescent

Sheffield S10 2BP

m.klonizakis@shu.ac.uk 


\section{ABSTRACT}

\section{Background}

Preserving endothelial function and microvascular integrity is suggested to reduce cardiovascular disease risk. It was recently shown that the age-dependent decline in endothelial and microvascular integrity may be reversed when combining exercise with Mediterranean Diet (MD) in an eight-week intervention. The present study investigates whether the risk-reduction improvement in microcirculatory and cardiorespiratory functions are sustained in this age-group after a one-year follow-up.

\section{Design and Methods}

Twenty sedentary healthy participants (age, $55 \pm 4$ years) from the original study, underwent cardiopulmonary exercise tolerance test, and were assessed for their upper- and lower-limb vascular endothelial Cutaneous Vascular Conductance (CVC) using laser Doppler fluximetry (LDF) with endothelium-dependent [ACh (acetylcholine chloride)] and -independent [SNP (sodium nitroprusside)] vasodilation, one year after completing the intervention.

\section{Results}

Both MD- and exercise-group appeared to have an improved microvascular responses, in comparison to baseline as far as ACh is concerned. Exploring the interactions between the timepoint and the original group however, revealed a stronger improvement in the MD-group in comparison to the exercise-group, for $\operatorname{ACh}(P=0.04, d=0.41)$. In the upper-body, the time-point and group interaction for $\mathrm{ACh}$, indicated a better improvement for $\mathrm{MD}$, without however statistical significance $(P=0.07, d=0.24)$. Additionally, cardiorespiratory improvement in Ventilatory Threshold was maintained, one year after (12.2 \pm 3.0 vs. $\left.13.2 \pm 3.2 \mathrm{ml} \cdot \mathrm{kg}^{-1} \cdot \mathrm{min}^{-1}, \mathrm{p}<0.05\right)$. 


\section{Conclusions}

The original improvements from an 8-week exercise and MD intervention were still evident, particularly in the microcirculatory and cardiorespiratory- assessments, one year after the initial study. This suggests that a brief intervention combining MD with exercise in this high-risk group promises long-term health benefits.

Abstract word count: 250 


\section{INTRODUCTION}

Maintaining microvascular integrity is a crucial element of our health and wellbeing and is affected by the ageing process (Gates et al., 2009). A healthy microcirculatory system cannot only affect tissue viability and susceptibility but could potentially play a predictive role in disease generation and progression (Kahaleh, 2008; Khan et al., 2010; Klonizakis et al., 2003).

The relationship between the attenuation of skin microvascular, vasodilatory responses and aging alone is well documented (Gates et al., 2009; Tew et al., 2010), being largely the result of a depleted endothelial function (Gates et al., 2009). A number of studies (i.e. Dod et al., 2010; Versari et al., 2009) have exemplified the importance of maintaining endothelial function unaltered, as endothelium appears to be an early and important promoter for atherosclerosis and thrombosis, playing a decisive role in the occurrence of cardiovascular events.

With the occurrence of cardiovascular disease being on the rise (OECD, 2013) and subsequently the disease management cost adding a huge burden to healthcare systems around the globe (i.e. more than $£ 28$ billion in the UK alone; Luengo-Fernández, 2006); it is important to find appropriate strategies to stimulate disease reduction. It seems to be a poignant time to invest in strategies which reduce the risk of cardiovascular risk within a growing population of middle- to older-aged people in the Western world and in the UK in specific (ONS, 2012).

An emerging body of evidence suggests moderate-to-vigorous physical activity can reduce the risk of many chronic conditions, including cardiovascular diseases [Hamer and Stamatakis 2009; Klonizakis et al., 2012; Warburton et al., 2006). Exercise however, can only respond partially to the problem as it is widely agreed that our health is the result of a continuous interaction between a 
host of potential influences (Barton and Grant, 2006; Marmot et al., 2012) including (but not limited too) genetic, environmental and social factors. Although exercise on its own can beneficial, other lifestyle factors such as nutrition can play an important role as well, positively affecting our physical wellbeing throughout life (Kumanyika et al., 2012).

A number of healthy diets have been recently proposed to benefit health, including the Japanese (Willcox and Willcox, 2014), Nordic (Mithril et al., 2012) and Mediterranean (Bach-Faig et al., 2011) diets. The Mediterranean Diet [MD] has being particularly highlighted for its purported positive cardiac health effects and the potential to reduce chronic disease development (Estruch et al., 2013). Adherence to the MD [which is based on olive oil, fruit, vegetables and salad, fish, legumes, wholegrain foods, wine and limited consumption of red meat] has been associated with a significant improvement in health status as seen by a reduction in overall mortality and deaths due to cardiovascular causes; $9 \%$ for both (Sofi et al., 2008).

Considering the scientific evidence, it makes sense to attempt and promote the combined consumption of a healthy diet that has been proven to provide cardiovascular effects with exercise, focusing in the small veins of our body, due to their important role in our overall wellbeing. With the number of studies attempting this being limited (i.e. Lucas et al., 1987, Montero et al., 2012), it was recently attempted to bridge the knowledge gap; investigating the medium-term effects of an intervention that combined a lower-limb exercise training [in the form of treadmill-based exercise] and the MD, on both lower- and upper-limb cutaneous microvascular functions in a healthy, older population (Klonizakis et al., 2012). The original study attempted to create equilibrium between internal and external validity with a pragmatic approach. Firstly, we avoided implementing strict and full adherence to the MD, opting for a flexible educational and motivational method to 
promote consumption of the MD in a small sample of middle to older aged adults who resided in the UK. This age-group had little experience with this type of eating pattern and had no cultural or traditional connection to such a diet. Thus, we anticipated any strict adherence protocol as a challenging prospect for the participants. Hence, we implemented individual advisory and tailored sessions to prepare the participants to change current dietary habits. During those we encouraged the increase in several items of food and drink, which are strongly associated with beneficial health effects (vegetables, fruits, olive oil, tree nuts and fresh oily fish). When combining exercise with $M D$, we relied on allowing the participants to select from a range of effective intensities within the mild-to-moderate intensity domains, which allowed an excellent (approx. 90\%) compliance throughout the 8 weeks in both MD and non-MD groups. This pragmatic approach resulted in short-term effectiveness in reversing the age-dependent decline in exercise tolerance and improving microcirculatory endothelial function, especially in the MD-group (which combined MD and exercise) (Klonizakis et al., 2012). However, the longer-term compliance that is needed, in order to sustain these cardiovascular risk-reduction benefits, is unknown.

This investigation follows up from the original data recorded for lower- and upper- limb cutaneous microvascular and cardiorespiratory assessments one year after the end of the original study (Klonizakis et al., 2012). The aim of this study was to examine if the participants retained any of the original microcirculatory and cardiovascular benefits in this time-frame.

\section{METHODS}

Twenty out of 22 participants of the original study ( 2 dropped out due to relocation), returned for this follow-up study after 1 year since they completed the original intervention. Since then, participants received no instruction or support regarding exercise and/or the MD. The 
consumption of MD wasn't reported by any of the participants in either groups at the time of the follow-up measurements.

No reason for exclusion existed for any other of the original cohort and no change in participants' health status (Table 1). None of the participants was smoking or receiving $\beta$-blockers at the time of their follow-up assessment. Participants were asked to refrain from any regular or structured exercise activities for 48 hours and to abstain from caffeine for 24 hours prior to the measurement session. This study gained institutional ethical approval and was carried out in accordance with the Declaration of Helsinki of the World Medical Association.

\section{Assessment of cutaneous microvascular function}

All microvascular assessments were performed in a temperature-controlled room [range, $22-24{ }^{\circ} \mathrm{C}$ ] following a 10-min acclimatization period.

The Laser Doppler Fluximetry measurements [moorVMS-LDF2, Moor Instruments] involved an incremental dose-response iontophorosis protocol (Klonizakis et al., 2012) using two Perpex probes with $80 \mu \mathrm{l}$ of the endothelium-dependent, ACh [Miochol-E; Novartis] and endotheliumindependent SNP [sodium nitroprusside; Rottapharm, S.L.] vasodilators. All participants were assessed for both lower and uppers body in supine position as previously described (Klonizakis et al., 2003; Klonizakis et al., 2012).

\section{Cardiopulmonary exercise assessment}

Following a 15-minute rest, participants followed an incremental exercise test on a treadmill [Cosmos HP Mercury 5.0, Nussdorf-Traunstein / Germany] with velocity initiated at $2.0 \mathrm{~km} / \mathrm{h}$ and was increased by $1.0 \mathrm{~km} / \mathrm{h}$ every 3 min until reaching the test termination criteria as previously 
stated (Balady et al., 2010) Cardiopulmonary responses of $\dot{\mathrm{VO}}_{2}$ [oxygen uptake], $\dot{\mathrm{VCO}}_{2}\left[\mathrm{CO}_{2}\right.$ production] and RER [respiratory exchange ratio] were continuously measured breath-by-breath, using an online gas analyser [Metalyzer Cortex 3B]. Flow sensor and gas analysers using gases of known concentration [ $16 \%$ for $\mathrm{O}_{2}$ and $5 \%$ for $\mathrm{CO}_{2}$ ] and a $3 \mathrm{~L}$ gas volume syringe were calibrated prior to each test.

\section{Data recording and analysis}

Microvascular, endothelium-dependent and -independent functions were measured via peak cutaneous flux responses to ACh and SNP, recorded in conventional PU (perfusion units) (Klonizakis et al., 2012). Tmax (time to reach maximum perfusion) was also measured (Klonizakis et al., 2011).

Cutaneous blood flux data were also divided by mean arterial pressure to calculate CVC (cutaneous vascular conductance). Differences in group characteristics were assessed using independent Student's $t$ tests and $\chi^{2}$ tests. Mixed-model (group by time), ANCOVA (analysis of covariance) were used to detect changes in outcome measures between groups, with baseline data used as the covariate. Effect sizes (Cohen's $d$ ) were calculated for the exercise group data, with 0.2 , 0.5 and 0.8 representing small, medium and large effects respectively. Cardiorespiratory $\dot{\mathrm{V}}_{2}$ and $\dot{\mathrm{VCO}}_{2}$ data were averaged for the final minute of each stage, while ventilatory threshold (VT) was determined individually for each test using the V-slope method as previously described (Klonizakis et al., 2012). Two-way mixed ANOVA model was applied to detect the intervention effects on VT, with training (before and after) as within subjects. Statistical significance was set at $P \leq 0.05$. 


\section{RESULTS}

\section{Perfusion in lower limbs}

Both the MD and the exercise group appeared to have an improved raw CVC, 1 year after the intervention, in comparison to the baseline measurements, and as far as ACh is concerned (e.g. $P=0.03, d=0.55)$. This was not the case with SNP $(P=0.3, d=0.36)$. The previously-reported interaction between the time-point (e.g. prior, 8 weeks and 1-year following the intervention) and the dose delivered (e.g. $250 \mu \mathrm{Cb}, 500 \mu \mathrm{Cb}, 1000 \mu \mathrm{Cb}$ and $2000 \mu \mathrm{Cb}$ ) was persistent for ACh $(P=0.04, d=0.46$; Figure $1 \mathrm{a}$ and $1 \mathrm{~b}$ for MD and exercise group respectively), but not for SNP $(P=0.26, d=0.20)$. Exploring the interactions between the time-point and the original group (e.g. $M D$ and exercise vs exercise only) however, revealed a stronger improvement in the MD group in comparison to the exercise group, for $\mathrm{ACh}$, following the intervention ( $P=0.04, d=0.51$; Figure 2$)$. No significant differences was observed between the MD group and the exercise group in endothelium-independent vasodilation (SNP), 1-year period after the intervention $(P=0.7, d=0.1)$.

In regards to the situation for the endothelial-dependent (ACh) and-independent (SNP) vasodilation, between the $2^{\text {nd }}$ time-point of the intervention ( 8 weeks) and 1 year after the completion of the intervention, our findings suggest that no statistical significant differences exist, either in regards to the time-point (e.g. $P=0.06, d=0.31$ for ACh and $P=0.32, d=0.46$ for SNP) or for the time-point - group interactions (e.g. $P=0.3, d=0.36$ for ACh and $P=0.27, d=0.51$ for SNP).

\section{Perfusion in upper limbs}

In comparison to the baseline measurements, both groups (e.g. MD group and the exercise group) appeared to have an improved raw CVC, 1 year after the completion of the intervention as far as ACh (e.g. $P=0.001, d=0.55)$ and SNP are concerned $(P=0.04, d=0.3$; Table 2). 
The previously-reported interaction between the time-point and the dose delivered was persistent for ACh ( $P=0.001, d=0.49)$, with no differences being detected for SNP $(P=0.54, d=0.12)$. The timepoint and group interaction interactions for ACh showed a better improvement for the MD, without however reaching overall statistical significance despite being close $(P=0.07, d=0.34$; Table 2). Similarly, results for endothelium-independent vasodilation (SNP), following the 1-year period, showed no overall statistical significance $(P=0.11, d=0.27)$, although differences were observed at individual doses (e.g. $1000 \mu \mathrm{Cb}$; Table 2).

In a similar manner to what it was observed in the lower limbs, the situation remained unchanged between the 8-week and 1 year time-points for both ACh and SNP, for the time-point (e.g. $P=0.81$, $d=0.33$ for ACh and $P=0.25, d=0.26$ for SNP) and for the time-point - group (e.g. MD or exercise group) interactions (e.g. $P=0.41, d=0.29$ for ACh and $P=0.65, d=0.30$ for SNP).

\section{Tmax}

In agreement to our 8-week findings, no statistically-significant differences were observed either between groups (e.g. 179.1 \pm 22.3 for the exercise group compared with $181.6 \pm 22.0$; for the MD group; $P=0.75$ at $2000 \mu \mathrm{Cb}$ ACh, 1 year after the intervention) or between assessments (e.g. 182.4 \pm 25.1 compared with $179.1 \pm 22.3 ; P=0.6$ at $2000 \mu \mathrm{Cb}$, for $\mathrm{ACh}$ in the Exercise group), for both agents. Similarly no interactions were observed between time-point and dose of the agent $(P=0.40$, $d=0.11$ for ACh; and $P=0.55, d=0.09$ for SNP). 
The initial intervention-dependent cardiorespiratory improvement in VT $(12.2 \pm 3.0$ vs. $15.1 \pm 3.1$ $\left.\mathrm{ml} . \mathrm{kg}^{-1} \cdot \mathrm{min}^{-1}, \mathrm{p}<0.01\right)$, was maintained after one year compared with baseline $(12.2 \pm 3.0 \mathrm{vs}$. 13.2 $\left.\pm 3.2 \mathrm{ml} . \mathrm{kg}^{-1} \cdot \mathrm{min}^{-1}, \mathrm{p}<0.05\right)$ with no significant deterioration in the follow-up compared with the post intervention stage. However, no interaction was found between MD group and exercisegroup. Within groups comparisons showed a trend, though not significant, towards sustained benefits of the MD group (Figure 3).

\section{DISCUSSION}

Acting in response to the growing evidence supporting the MD [26] as a healthy diet for CVD riskreduction, it was recently explored the effects of an intervention combining exercise with the introduction of a "healthy" diet in the participants' lifestyle (Klonizakis et al., 2012). The study results were more than encouraging, suggesting improvements in endothelial-dependent microvascular function and cardiopulmonary fitness in general and in the MD group in particular (Klonizakis et al., 2012).

The findings of our current study follow the same trend, being overly positive: we observed that the effects persisted 1 year after the end of the intervention, with endothelial function remaining improved in the MD group, when compared to the initial assessments (e.g. relative increases ranging between $74-100 \%$, between visits for ACh at different doses in the lower limb) with marked differences existing in the lower limbs and positive signs existing for the upper body as well. We also noted that positive effects remained statistically unchanged between the end of the intervention and 1 year after. This suggests that although the effect was not as strong as initially observed, the positive influence of the intervention persisted being in agreement with studies that 
followed epidemiologically MD consumers for a longer period of time (Sofi et al., 2008). This shows that an intervention combining exercise and MD, offers longer-term benefits taking into consideration the positive implications associated with MD-consumption (Estruch et al., 2013; Grosso et al., 2014).

Attributing those findings could be a challenging task. As an almost equal number of participants in both groups continued to take part in exercise activities following the intervention completion, the possibility that the persistence of the positive differences is accounted to exercise only can be excluded, although they will undoubtedly play a positive role as it is well-established (Klonizakis et al., 2012; Tew et al., 2010; Warburton et al., 2006]. Therefore, It may be that changes occurring at a molecular level due to MD (e.g. in the serum markers of endothelial dysfunction/adhesion molecule, or/and in the inflammatory markers [CRP (C-reactive protein)] or angiogenesis) (Dod et al., 2010) which have been particularly attributed to olive oil and tree nuts (Urpi-Sarda et al., 2012) last longer than expected. Indeed, it has been suggested that the olive oil compounds (such as oleic acid, phytosterols ( $\beta$ - sitosterol), antioxidants ( $\alpha$-tocopherol) and phenolic compounds) offer longer-term benefits as far as anti-inflammation and endothelial function are concerned (Perona et al., 2006); hence, it is possible that this, together with the proven benefits of exercise for this agegroup (Tew et al., 2010) are responsible for our observations. However, further studies (that will be adequately-and specifically-powered) are required for this result to be substantiated. For example, it will be worth to explore the differences of our current, unsupervised, follow-up approach with a more structured one that will assess participants' MD adherence over a longer time-period or explore specific blood biomarkers at different time-points, which would allow attributing findings to specific intervention effects. 
Finally, the fact that there was no deterioration in the cardiorespiratory capacity is encouraging. This could reflect sustained adaptation in age-dependent musculoskeletal, hormonal and circulatory functions that are well established for exercise (ACSM et al., 2009) and conforms with recent findings about the MD benefits indicated by improvement in several metabolic biomarkers (Richard et al., 2013). Longitudinal MD adherence studies have now confirmed MD as a primary CVD-prevention mechanism (Estruch et al., 2013) and CVD risk-reduction benefits in this age-group [21]. Therefore, it is no surprise that combining MD with exercise could further reduce the risk in this high-risk (for CVD) group. However, it remains difficult to distinguish the sustained MD benefits alone without considering the exercise-dependent adaptations in the present study.

\section{CONCLUSIONS}

Long term reduction in cardiovascular disease risk through either exercise or diet is a common challenge for intervention studies, particularly when introducing a relatively unfamiliar dietary patterns and exercise to high-risk age-groups. Our combined approach of MD and exercise seems to provide a promising sustained long-term improvement in endothelial microvascular and cardiorespiratory functions. Nevertheless, it remains difficult to attribute any risk-reduction benefits to exercise or diet alone. Future studies may confirm the long-term risk-reduction outcomes that are specifically associated with MD-adherence such as pro-/anti-inflammatory and lipid-profile markers (Estruch et al., 2013; Lucas et al., 1987; Montero et al., 2012).

\section{ACKNOWLEDGMENTS}

The authors would like to thank Dr. Mark Smith for his work on the original study. This study has been supported financially by of the College of Social Science of the University of Lincoln. 


\section{REFERENCES}

American College of Sports Medicine, et al., 2009. American College of Sports Medicine position stand. Exercise and physical activity for older adults. Med. Sci. Sports Exerc. 41, 1510-30.

Bach-Faig, A., et al., 2011. Mediterranean diet pyramid today. Science and cultural updates. Public Health Nutr. 14, 2274-84.

Balady, G.J., et al., 2010. Clinician's Guide to cardiopulmonary exercise testing in adults: a scientific statement from the American Heart Association. Circulation 122, 191-225.

Barton, H., Grant, M., 2006. A health map for the local human habitat. J. R. Soc. Promot. Health 126, 252-3.

Dod, H.S., et al., 2010. Effect of intensive lifestyle changes on endothelial function and on inflammatory markers of atherosclerosis. Am. J. Cardiol. 105, 362-7.

Estruch, R., et al., 2013. Primary prevention of cardiovascular disease with a Mediterranean diet. N. Engl. J. Med. 368, 1279-90.

Gates, P.E., et al., 2009. Human endothelial function and microvascular ageing. Exp. Physiol. $94,311-16$

Grosso, G., et al., 2014. Mediterranean diet and cardiovascular risk factors: A systematic review. Crit. Rev. Food Sci. Nutr. 54, 593-610.

Hamer, M., Stamatakis, E., 2009. Physical activity and risk of cardiovascular disease events: inflammatory and metabolic mechanisms. Med. Sci. Sports Exerc. 41, 1206-11.

Kahaleh, B., 2008. Vascular disease in scleroderma: Mechanisms of vascular injury. Rheum. Dis. Clin. North Am. 34, 57-71.

Khan, F., et al., 2010. The role of endothelial function and its assessment in rheumatoid arthritis. Nat. Rev. Rheumatol. 6, 253-61. 
Klonizakis, M., et al., 2003. Effects of Posture and Venous Insufficiency on Endothelial-

Dependent and -Independent Cutaneous Vasodilation in the Perimalleolar Area. Eur. J. Vasc. Endovasc. Surg. 26, 100-4.

Klonizakis, M., et al., 2011. Characterising the time-course of microvascular vasodilator responses in humans using laser Doppler fluximetry and Iontophoresis. J. Pharmacol. Toxicol. Methods 63, 115-8.

Klonizakis, M., et al., 2012. Mediterranean diet and exercise induce improvement in agedependent vascular activity. Clin. Sci. 124, 579-87.

Kumanyika, S., 2012. Preventive medicine and diet-related diseases: Searching for impact. Prevent. Med. 55, 542-3.

Lucas, C.P., et al., 1987. Achieving therapeutic goals in insulin-using diabetic patients with noninsulin-dependent diabetes mellitus. A weight reduction-exercise-oral agent approach. Am. J. Med. 83, 3-9.

Luengo-Fernández, R., et al., 2006. Cost of cardiovascular diseases in the United Kingdom. Heart 92, 1384-9.

Marmot, M., et al., 2012. WHO European review of social determinants of health and the health divide. Lancet 380, 1011-29.

Mithril, C., et al., 2012. Guidelines for the new Nordic diet. Public Health Nutr. 15, 1941-47. Montero, D., et al., 2012. Endothelial dysfunction, inflammation, and oxidative stress in obese children and adolescents: markers and effect of lifestyle intervention. Obes. Rev. 13, 44155.

Office of National Statistics, 2012. Population Ageing in the United Kingdom, its Constituent Countries and the European Union. Available at: www.ons.gov.uk/ons/rel/mortality- 
ageing/focus-on-older-people/population-ageing-in-the-united-kingdom-and-europe/rpt-

age-uk-eu.html (last accessed 29 July 2014).

Organisation for Economic Co-operation and Development, 2013. Health at a Glance 2013:

OECD Indicators. Available at: http://www.oecd.org/els/health-systems/Health-at-a-Glance2013.pdf (last accessed 29 July 2014).

Perona, J.S., et al., 2006. The role of virgin olive oil components in the modulation of endothelial function. J. Nutr. Biochem. 17, 429-45.

Richard, C., et al., 2013. Effect of the Mediterranean diet with and without weight loss on markers of inflammation in men with metabolic syndrome. Obesity $21,51-7$.

Sofi, F., et al., 2008. Adherence to Mediterranean diet and health status: meta-analysis. Br. Med. J. 337, 1344-51.

Tew, G.A., et al., 2010. Effects of ageing and fitness on skin-microvessel vasodilator function in humans. Eur. J. Appl. Physiol. 109, 173-81.

Urpi-Sarda, M., et al., 2012. Virgin olive oil and nuts as key foods of the Mediterranean diet effects on inflammatory biomakers related to atherosclerosis. Pharmacol. Res. 65, 577-83. Versari, D., et al., 2009. Endothelium-dependent contractions and endothelial dysfunction in human hypertension. Br. J. Pharmacol. 157, 527-36.

Warburton, D.E., et al., 2006. Prescribing exercise as preventive therapy. Can. Med. Assoc. J. $174,961-74$.

Willcox, B.J., Willcox, D.C., 2014. Caloric restriction, caloric restriction mimetics, and healthy aging in Okinawa: controversies and clinical implications. Curr. Opin. Clin. Nutr. Metab. Care $17,51-58$ 
Table 1: Demographics of Participants

Values are means \pm S.D.

\begin{tabular}{|c|c|c|c|}
\hline Variable & Exercise Group & $\begin{array}{c}\text { Mediterranean Diet } \\
\text { Group }\end{array}$ & $P$ value \\
\hline $\begin{array}{l}\text { Gender }(n) \\
\text { (female/male) }\end{array}$ & $7 / 3$ & $7 / 3$ & 1.00 \\
\hline Age (year) & $57 \pm 4$ & $56 \pm 4$ & 0.85 \\
\hline Body mass (kg) & $75.2 \pm 17.3$ & $76.9 \pm 13.9$ & 0.44 \\
\hline Stature $(\mathrm{cm})$ & $165.8 \pm 9.9$ & $167.4 \pm 7.7$ & 0.88 \\
\hline $\begin{array}{l}\text { MAP (mean arterial } \\
\text { pressure) }(\mathrm{mmHg})\end{array}$ & $96.1 \pm 14.4$ & $94.6 \pm 12.5$ & 0.61 \\
\hline $\begin{array}{l}\text { Systolic pressure } \\
(\mathrm{mmHg})\end{array}$ & $124.4 \pm 21.5$ & $125.5 \pm 11.0$ & 0.69 \\
\hline $\begin{array}{l}\text { Diastolic pressure } \\
(\mathrm{mmHg})\end{array}$ & $79.3 \pm 10.5$ & $77.5 \pm 10.9$ & 0.61 \\
\hline $\begin{array}{l}\text { Regular Exercise } \\
\text { (yes/no) }\end{array}$ & $7 / 3$ & $5 / 5$ & 0.39 \\
\hline
\end{tabular}


Table 2: Raw CVC comparison between groups for ACh and SNP in the upper limbs Values are means $\pm S$.D.

\begin{tabular}{|c|c|c|c|c|c|c|}
\hline \multirow[t]{2}{*}{$\begin{array}{l}\text { Dose } \\
\text { (ACh) }\end{array}$} & \multicolumn{2}{|c|}{ Exercise Group } & \multicolumn{2}{|c|}{ Mediterranean diet Group } & \multicolumn{2}{|c|}{$\begin{array}{c}\text { P-value } \\
\text { (Between } \\
\text { Groups-BG) }\end{array}$} \\
\hline & Before & 1-year after & Before & $\begin{array}{c}\text { 1-year } \\
\text { after }\end{array}$ & Before & $\begin{array}{c}\text { 1-year } \\
\text { after }\end{array}$ \\
\hline Baseline & $0.22 \pm 0.06$ & $0.21 \pm 0.05$ & $0.25 \pm 0.05$ & $0.22 \pm 0.11$ & 0.76 & 0.67 \\
\hline $250 \mu \mathrm{Cb}$ & $0.29 \pm 0.09$ & $0.34 \pm 0.16$ & $0.33 \pm 0.07$ & $0.50 \pm 0.35$ & 0.39 & 0.41 \\
\hline $500 \mu \mathrm{Cb}$ & $0.38 \pm 0.13$ & $0.60 \pm 0.34$ & $0.31 \pm 0.12$ & $1.05 \pm 1.03$ & 0.31 & 0.04 \\
\hline $1000 \mu \mathrm{Cb}$ & $0.47 \pm 0.26$ & $0.98 \pm 0.38$ & $0.42 \pm 0.19$ & $1.46 \pm 1.34$ & 0.57 & 0.07 \\
\hline $2000 \mu \mathrm{Cb}$ & $0.86 \pm 0.44$ & $1.89 \pm 0.57$ & $0.74 \pm 0.26$ & $2.22 \pm 1.1$ & 0.52 & 0.12 \\
\hline Dose & Exercis & Group & Mediterrane & diet Group & P-valu & (BG) \\
\hline (SNP) & Before & 1-year after & Before & $\begin{array}{c}\text { 1-year } \\
\text { after }\end{array}$ & Before & $\begin{array}{c}\text { 1-year } \\
\text { after }\end{array}$ \\
\hline Baseline & $0.21 \pm 0.08$ & $0.21 \pm 0.04$ & $0.25 \pm 0.05$ & $0.26 \pm 0.06$ & 0.38 & 0.34 \\
\hline $250 \mu \mathrm{Cb}$ & $0.30 \pm 0.14$ & $0.37 \pm 0.17$ & $0.40 \pm 0.12$ & $0.56 \pm 0.40$ & 0.12 & 0.11 \\
\hline $500 \mu \mathrm{Cb}$ & $0.41 \pm 0.21$ & $0.57 \pm 0.19$ & $0.48 \pm 0.21$ & $0.88 \pm 0.70$ & 0.56 & 0.07 \\
\hline $1000 \mu \mathrm{Cb}$ & $0.65 \pm 0.40$ & $0.98 \pm 0.62$ & $0.59 \pm 0.31$ & $1.47 \pm 0.92$ & 0.69 & 0.04 \\
\hline $2000 \mu \mathrm{Cb}$ & $1.16 \pm 0.89$ & $1.82 \pm 1.22$ & $0.84 \pm 0.36$ & $2.32 \pm 0.81$ & 0.23 & 0.08 \\
\hline
\end{tabular}


Figure 1a: Microvascular comparison between time-points (baseline vs 1 year after the intervention) for CVC results in the MD group, for ACh in the lower limb. * $\mathrm{P}<0.05$.

Figure 1b: Microvascular comparison between time-points (baseline vs 1 year after the intervention) for CVC results in the exercise group, for ACh in the lower limb. ${ }^{*} \mathrm{P}<0.05$.

Figure 2: Microvascular comparison between groups for CVC results for ACh in the lower limb, 1 year after the intervention. ${ }^{*} \mathrm{P}<0.01,{ }^{*} \mathrm{P}<0.05$.

Figure 3: Ventilatory threshold comparison for the whole group (combined) at different time points, after eight weeks $(p<0.01)$ and after one year $(p<0.05)$ compared with baseline. 


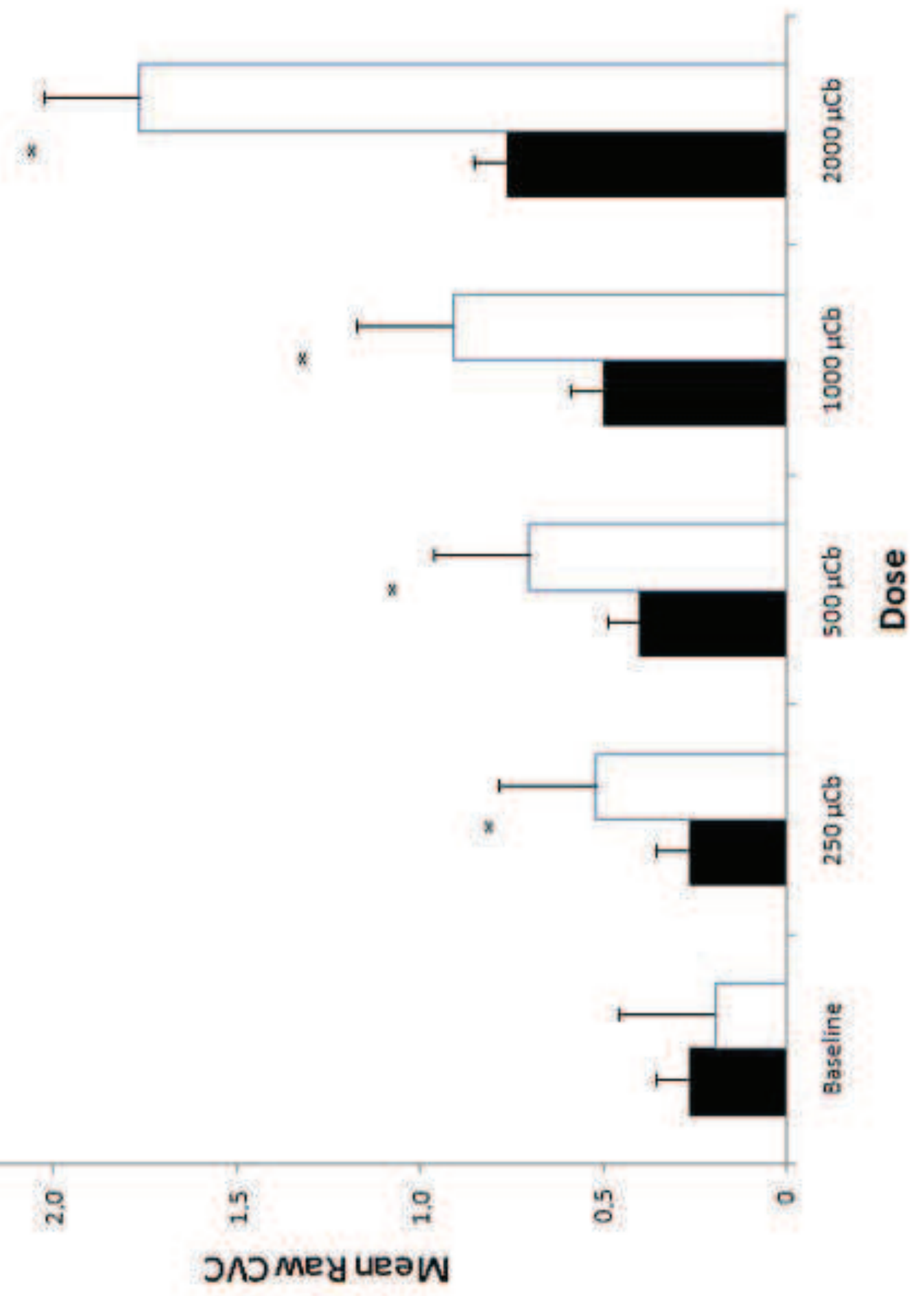

$\stackrel{0}{\circ}$

过

。․

政

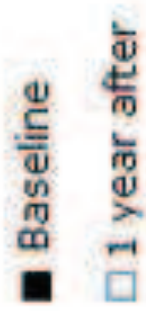

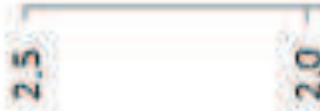

J 


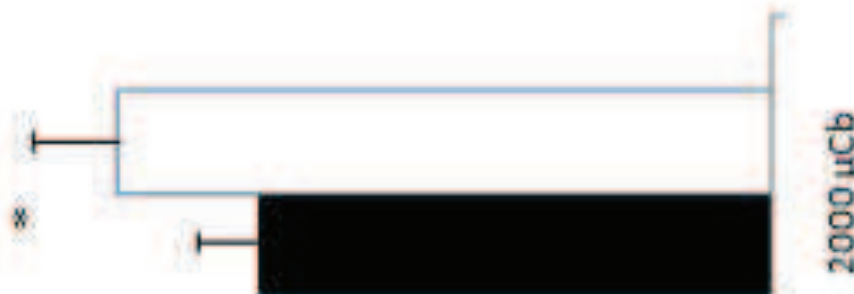

음

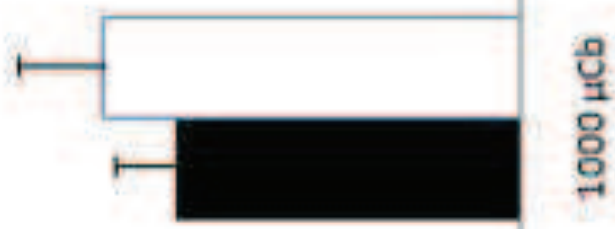

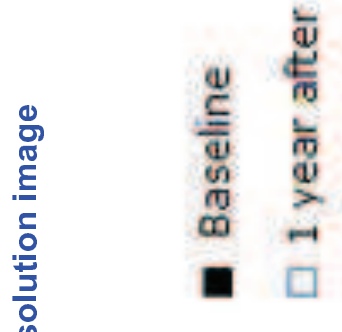

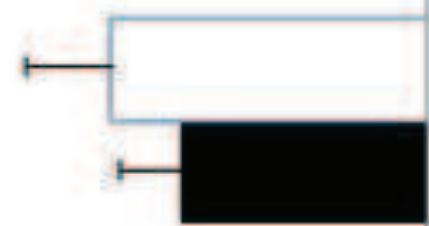

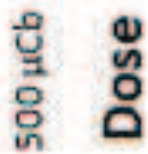

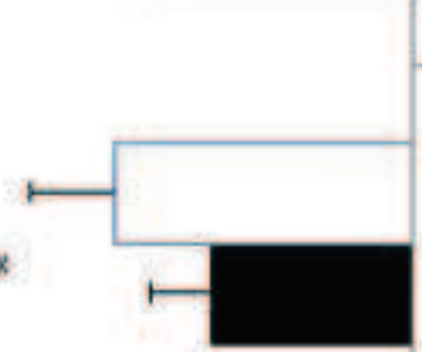

을

政 


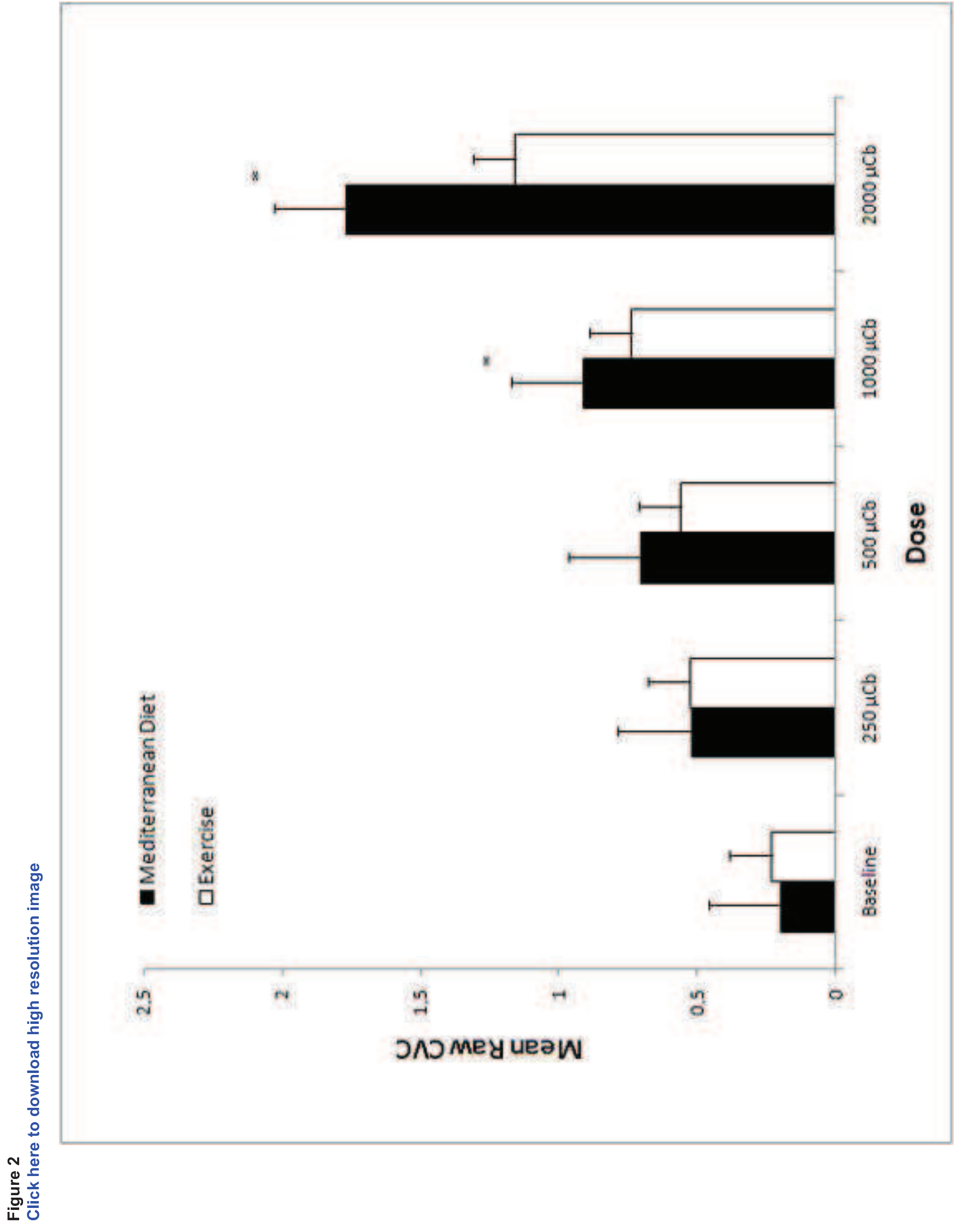




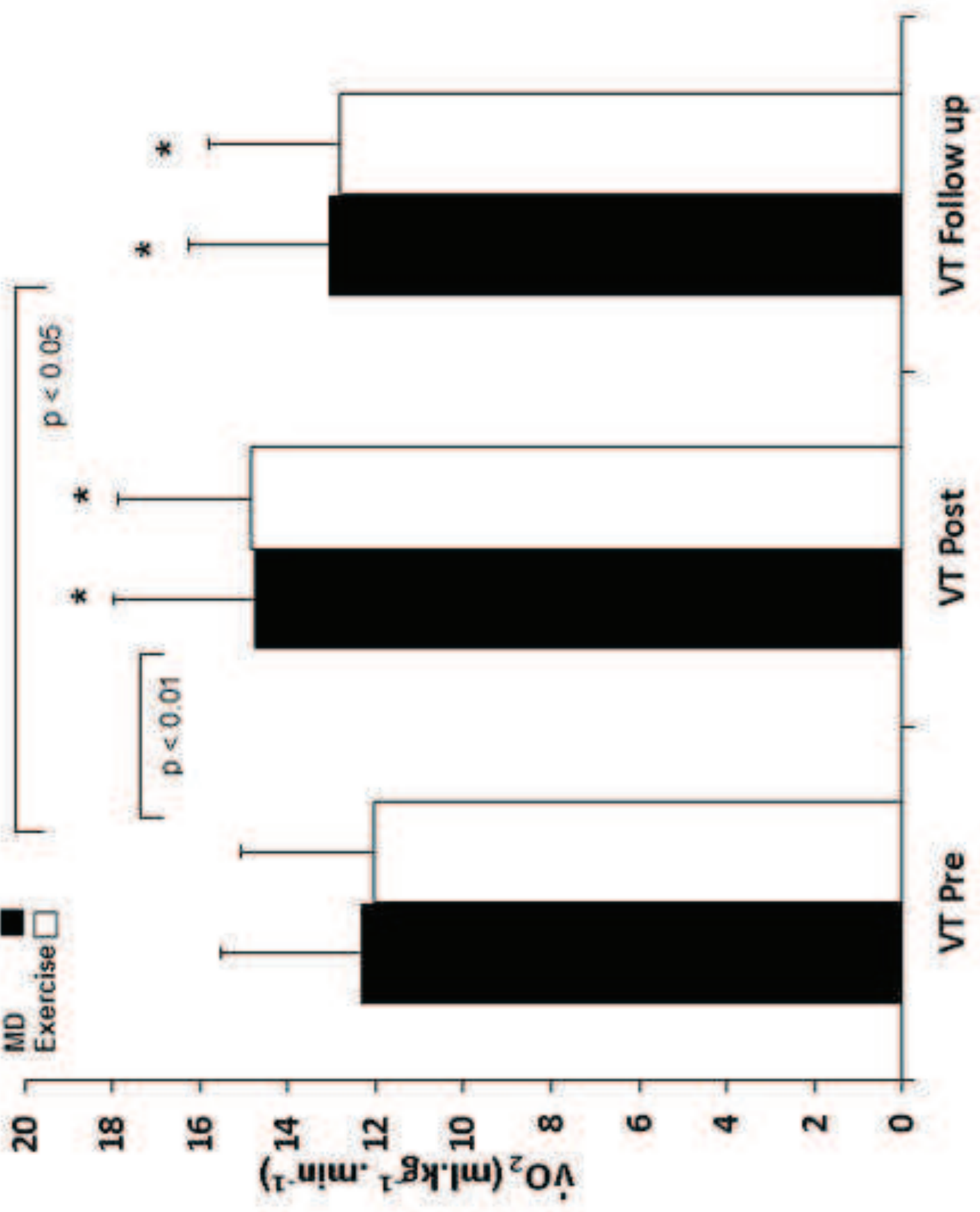

m

인

은 